\title{
Implementation of Cord- Blood Derived Unrestricted Somatic Stem Cells in the Regeneration of two Experimental Models: Carbon Tetrachloride and S. Mansoni Induced Liver Fibrosis
}

\author{
Manal Kamel, Zeinab Demerdash, Hanan El-Baz, Salwa Hassan, Faten Salah, Wafaa Mansour, Olfat \\ Hamamm \\ Department of Immunology and Pathology, Theodor Bilharz Research Institute \\ Giza, Egypt \\ baher_ronny@hotmail.com; helbaz77@hotmail.com; zeinbdem@hotmail.com
}

\section{Extended Abstract}

Liver fibrosis is the wound-healing response of the liver to chronic injury it is very important to investigate different treatments and therapies for cirrhosis as the liver is one of the target organs for which stem cell-based therapeutics is very promising. In this study, Isolation, propagation, and characterization of unrestricted somatic stem cells (USSCs) from cord blood (CB) samples were performed and induced to differentiate into osteoblasts, adipocytes and hepatocyte-like cells. The therapeutic potentiality of USSCs in two experimental models of chronic liver injury was evaluated. First experimental model (30 mice): Ten Schistosoma mansoni infected mice were intravenously injected with USSCs $1 \times 10^{6}$ cell/mouse. Ten were infected untreated (pathological control) and 10 healthy mice (negative control). 2nd experimental model (30 hamsters): Twenty were injected with repeated doses of carbon tetrachloride Sigma-Aldrich Chemical Co. (St Louis, Missouri, USA) to induce liver fibrosis; 10 were treated with intrahepatic injection of $3 \times 10^{6}$ USSCs and the other 10 were untreated pathological control. Ten healthy hamsters served as negative control. Animals were sacrificed 12 weeks post transplantation, and their liver sections were examined for detection of human hepatocyte-like cells by immunohistochemical staining. Moreover, liver sections were examined for fibrosis levels. Sera of sacrificed animals were tested for liver functions. CB USSCs, with fibroblast-like morphology, expressed high levels of CD44, CD90, CD73 and CD105 and were negative for CD34, CD45, and HLA-DR. USSCs showed high expression of transcripts for Oct4 and Sox 2 and were in vitro differentiated into osteoblasts, adipocytes, and hepatocyte-like cells. In both models transplantation of CBUSSCs resulted in engraftment of the fibrosed livers with newly formed hepatocytes evidenced by positive immunostaining with human Hep Par1, $\alpha$-fetoprotein, CK-18, CK-7 and OV6. Transplanted liver sections showed diminished hepatic fibrosis with significantly lower fibrotic index as well as significantly improved liver functions compared to the pathological control $(\mathrm{p}<0.001)$.

\section{Conclusion}

These data provide hope that human CB- derived USSCs are introduced as multipotent stem cells with great potentiality in regenerative medicine \& strengthens the concept of cellular therapy for the treatment of liver fibrosis.

This work is extracted from the project 1410 supported by the Science and Technology Development Funds (STDF), Cairo, Egypt.

Keywords: Liver fibrosis, Unrestricted somatic stem cells, Cord blood, Carbon tetrachloride

\section{References}

[1] J. Li, L. Zhang, J. L. Xin, L. Jiang, T. Zhang, L. Jin, P. Zhou, S. Hao, H. Cao, and L. Li, "Immediate intraportal transplantation of human bone marrow mesenchymal stem cells prevents death from fulminant hepatic failure in pigs," Hepatology, vol. 56, no. 3, pp. 1044-105, 2012.

[2] R. L. Pan, L. X. Wang, and J. Z. Shao, "Delta-like 1 serves as a new target and contributor to liver fibrosis downregulated by mesenchymal stem cell transplantation," J. Biol. Chem., vol. 286, pp. 12340-12348, 2011.

[3] M. Maleki, F. Ghanbarvand, M. R. Behvarz, M. Ejtemaei, and E. Ghadirkhomi, "Comparison of Mesenchymal Stem Cell Markers in Multiple Human Adult Stem Cells," Int. J. Stem Cells. vol. 7, no. 2, pp. 118-126, 2014. 
[4] D. Li, J. Fan, X. He, X. Zhang, Z. Zhang, Z. Zeng, M. Ruan, and L. Cai "Therapeutic effect comparison of hepatocyte-like cells and bone marrow mesenchymal stem cells in acute liver failure of rats," Int. J. Clin. Exp. Pathol., vol. 8, no. 1, pp. 11-24, 2015.

[5] X. J. Liang, X. J. Chen, D. H. Yang, S. M.Huang, G. D. Sun, and Y. P. Chen, "Differentiation of human umbilical cord mesenchymal stem cells into hepatocyte-like cells by hTERT gene transfection in vitro," Cell. Biol. Int., vol. 36 no. 2, pp. 215-21, 2012. 